\title{
Immune selection, senescence and adjuvant
}

\author{
TANG Hong \\ Institute of Virology, Chinese Academy of Sciences, Wuhan 430071, China
}

Received April 6, 2013

Citation: $\quad$ Tang H. Immune selection, senescence and adjuvant. Sci China Life Sci, 2013, 56: 391, doi: 10.1007/s11427-013-4481-5

The series of mini-reviews in this issue highlight the progress and open questions in three important areas in immunology, namely impaired central tolerance, senescence of innate cells and the magic adjuvant effect by recombinant flagellin on mucosal vaccines.

Self-nonself discrimination is the central mission of immune system. The thymus is endowed with the unique ability to generate adaptive $T$ cells which are immune competent against nonself while remain inert to self, the latter termed central tolerance. Impaired central tolerance frequently leads to autoimmunity. Negative selection and generation of natural regulatory $\mathrm{T}$ cell (nTreg) are two major mechanisms for thymus to establish central tolerance, where medullary thymic epithelial cells (mTECs) play critical roles for both negative selection and nTreg generation. Shi and Zhu [1] summarize and discuss current understanding and open questions about how mTECs develop and exert the critical function. Better understanding of mTECs will contribute to the better intervention of autoimmune diseases and immune reconstitution during aging or after infection, chemotherapy or radiotherapy.

Pathogens invasion remains an important cause of morbidity and mortality in the aged individuals. This is in part a consequence of immunosenescence, the deterioration of the immune response associated with aging. It is used to believe that immunosenescence mainly affects the adaptive immune system. However, recent studies have shown that the function of the diverse cells of the innate immune system is also affected with aging. Ma and Fang [2] compare the differ- ence between ectromelia virus (ECTV) infection and West Nile virus (WNV) infection in aged mice models.

Defects in T cells play a key role in age-related vulnerability to WNV infection, while defective natural killer cell (NK) responses following ECTV infection result in high mortality rate in aged mice. Poor innate immune responses are likely to play an important role in some age-related viral diseases. Dysfunctions of NK cells, a main effector cell type in innate immunity, clearly have an impact in the host immune defenses to viral infections in the aged individuals.

Streptococcus mutans ( $S$. mutans) infection leads to dental caries, one of the most common chronic diseases globally. Numerous vaccine strategies have failed because of adversity to induce and maintain protective seroconversion in oral fluids. Yan [3] reviews strategies to boost the salivary IgA response, and development of a nasal-spray anti-caries mucosal vaccine. He also discusses mucosal adjuvants for achieving protective immunity at oral mucosal membranes and summarizes the progress in recombinant flagellin-based anti-caries vaccine study in his own laboratory. Promising prospects are outlined for development of a nasal-spray or nasal-drop anti-caries vaccine and some other novel mucosal vaccines in children.

1 Shi Y Y, Zhu M Z. Medullary thymic epithelial cells, the indispensable player in central tolerance. Sci China Life Sci, 2013, 56: 392-398

2 Ma Y C, Fang M. Immunosenescence and age-related viral diseases. Sci China Life Sci, 2013, 56: 399-405

3 Yan H M. Salivary IgA enhancement strategy for development of a nasal-spray anti-caries mucosal vaccine. Sci China Life Sci, 2013, 56: $406-413$

Open Access This article is distributed under the terms of the Creative Commons Attribution License which permits any use, distribution, and reproduction in any medium, provided the original author(s) and source are credited.

email: tangh@wh.iov.cn 